\title{
A Rare Case of Recurrence of Craniopharyngioma at Ectopic Site after Surgical Resection of Primary Tumor
}

\author{
${ }^{1}$ Department of Neurosurgery, Command Hospital, (Central \\ Command), Lucknow, Uttar Pradesh, India \\ 2Department of Radiodiagnosis \& Imaging, Command Hospital \\ (Central Command), Lucknow, Uttar Pradesh, India \\ ${ }^{3}$ Department of Pathology, Command, Hospital (Central Command), \\ Lucknow, Uttar Pradesh, India \\ ${ }^{4}$ Department of Neurosurgery, Command, Hospital (Southern \\ Command), Pune, Maharashtra, India \\ Indian J Neurosurg 2018;7:265-268
}

Subir Dey ${ }^{1} \quad$ Akhilesh Rao $^{2}$ Shilpa Sinha ${ }^{2}$ Nishant $^{3}$ Sanjay Kumar ${ }^{4}$

\begin{abstract}
Address for correspondence Sanjay Kumar, MCh, Department of Neurosurgery, Command Hospital (Southern Command), Pune 411040, Maharashtra, India (e-mail: paraeagles@gmail.com).
\end{abstract}

\begin{abstract}
Ectopic recurrence of craniopharyngiomas after surgical resection of primary lesion is extremely rare. In this article, the authors describe a case of a 12-year-old girl with ectopic recurrence of craniopharyngioma in an extra-axial location in the right frontal region, after an attempt to completely resect the lesion originally located in the sellar region. The patient was investigated radiologically, hormonally, and histopathologic confirmation was sought. During surgery, it is important to take extra care in remov-

Keywords

- craniopharyngioma

- ectopic

- recurrence ing the primary lesion in toto. In case of cystic tumors, it is very important to aspirate cystic fluid completely, before resecting the capsule to avoid rupture or spillage. Longterm clinical and radiologic follow-up is suggested as ectopic recurrence may occur at a very late stage.
\end{abstract}

\section{Introduction}

Although craniopharyngiomas are a benign histological entity, they have a high tendency to recur at the same site even after an attempt to resect completely. However, it can recur at ectopic sites very rarely. Tumor seeding along the surgical tract or along the cerebrospinal fluid (CSF) pathways has been reported, which can result in ectopic recurrence. There exists particularly high risk for cystic tumors. Before the present case, 60 ectopic recurrent craniopharyngiomas have been reported up until 2016. The frontal lobe is the most common site of ectopic recurrence ( 23 cases). ${ }^{1}$ In our case, recurrence was seen in an extra-axial parasagittal location that has not been reported so far. Protection of the whole surgical bed and cautious manipulation of the tumor is required to prevent seeding of tumor. It should be emphasized that long-term radiologic surveillance is very important, even in patients undergoing complete resection. ${ }^{2,3}$

\section{Case History}

A 12-year-old girl underwent right pterional craniotomy and gross total excision of craniopharyngioma occupying the sellar region with suprasellar extension in January 2014 (-Fig. 1). On histopathologic examination (HPE), it was proven to be adamantinomatous type of craniopharyngioma. Postoperatively, the patient received radiotherapy and was on hormonal replacement therapy as she developed panhypopituitarism. During her follow-up visits in October 2016, she complained of headache. Clinical evaluation did not reveal any visual deficits or any other focal deficits. A noncontrast computer tomographic scan of her brain $(\boldsymbol{- F i g . ~ 2 a )}$ revealed postcraniotomy status in the right frontal region with ventriculoperitoneal (VP) shunt in situ. A well-defined hypodense area with a linear hyperdense septum at its inferior aspect and irregular discontinuous peripheral hyperdense rim, in an extra-axial, parasagittal location in the right received

November 9, 2017

accepted

January 19, 2018

published online

November 1, 2018
DOI https://doi.org/

10.1055/s-0038-1670933.

ISSN 2277-954X.
(C)2018 Neurological Surgeons'

Society of India
License terms

(®) $\Theta \circledast$ 

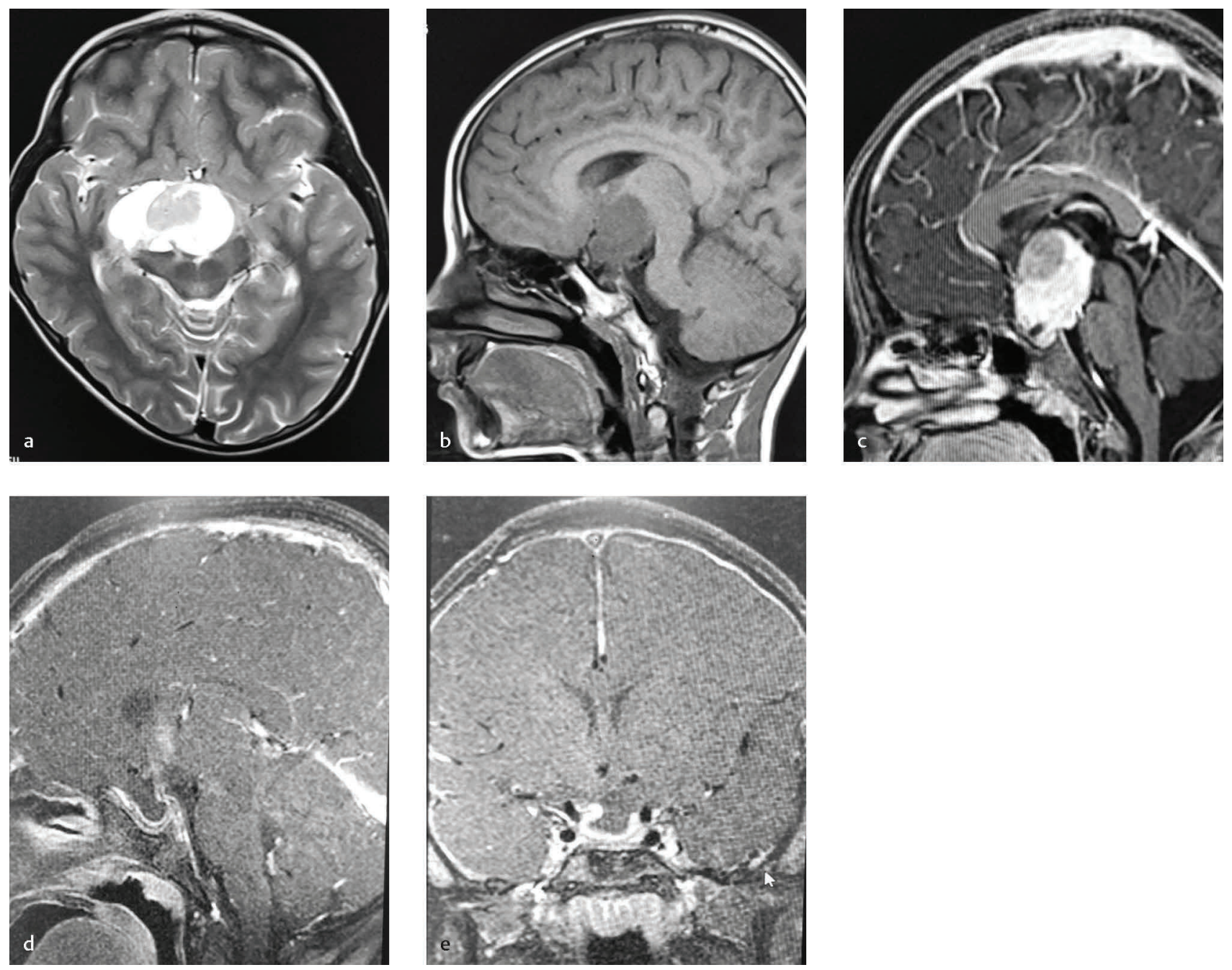

Fig. 1 (a) Preoperative T2 axial MRI showing heterogenous intensity lesion in sellar and suprasellar region. (b) Preoperative T1 saggital magnetic resonance hypointense lesion in sellar and suprasellar region. (c) Preoperative postcontrast saggital image showing enhancing solid component with cystic area. (d) Postoperative (first surgery) saggital image showing GTR of the tumor. (e) Postoperative (first surgery) coronal image showing GTR of tumor.

frontal region measuring approximately $43 \times 31 \times 41 \mathrm{~mm}$ in size was visualized. No significant mass effect was seen. Contrast-enhanced magnetic resonance imaging revealed a well-defined extra-axial lesion in the right frontal region in a parasagittal location broad-based toward falx cerebri along its anterior aspect. It was heterogeneously hyperintense on T2WI/FLAIR/T1WI (- Fig. 2b, c). Few thin septae were seen within. Mural thickening with altered signal intensity was seen in its anterior aspect suggestive of solid component, which revealed postcontrast enhancement (-Fig. 2d). Areas of magnetic susceptibility were seen within the lesion suggestive of calcification/hemorrhagic products. Medially, the lesion was causing mild contra lateral displacement of the falx cerebri. A midline shift of $7 \mathrm{~mm}$ was seen. On all other sides, it was causing compression of the brain parenchyma with mild displacement of frontal horn of the right lateral ventricle. Widening of sella with poor visualization of the pituitary gland was seen. The patient underwent redo craniotomy and resection of the lesion (-Fig. 2e). HPE of the resected lesion confirmed craniopharyngioma, and complex squamous epithelium and stellate reticulum were seen. Microcyst and wet keratin were present with fibrous stroma (-Fig. 2f). Postoperative recovery was uneventful. She was continued on hormonal replacement therapy, and after 1-year follow-up, there has been no recurrence of the lesion.

\section{Discussion}

Craniopharyngiomas are partially cystic tumors of the sellar region thought to arise from remnants of Rathke's pouch and craniopharyngeal duct, and are benign lesions per se. However, they can behave aggressively. It can spread to the adjacent areas to involve important structures around the sellar region due to which complete removal of the lesion may be very difficult. Tumor cells may remain behind if resection is not performed adequately and can give rise to recurrence of tumor at local as well as ectopic sites. ${ }^{1,4}$

Although recurrence at local site is common after gross total resection (GTR) of craniopharyngiomas, ectopic recurrence occurs very rarely. The incidence of ectopic recurrence 

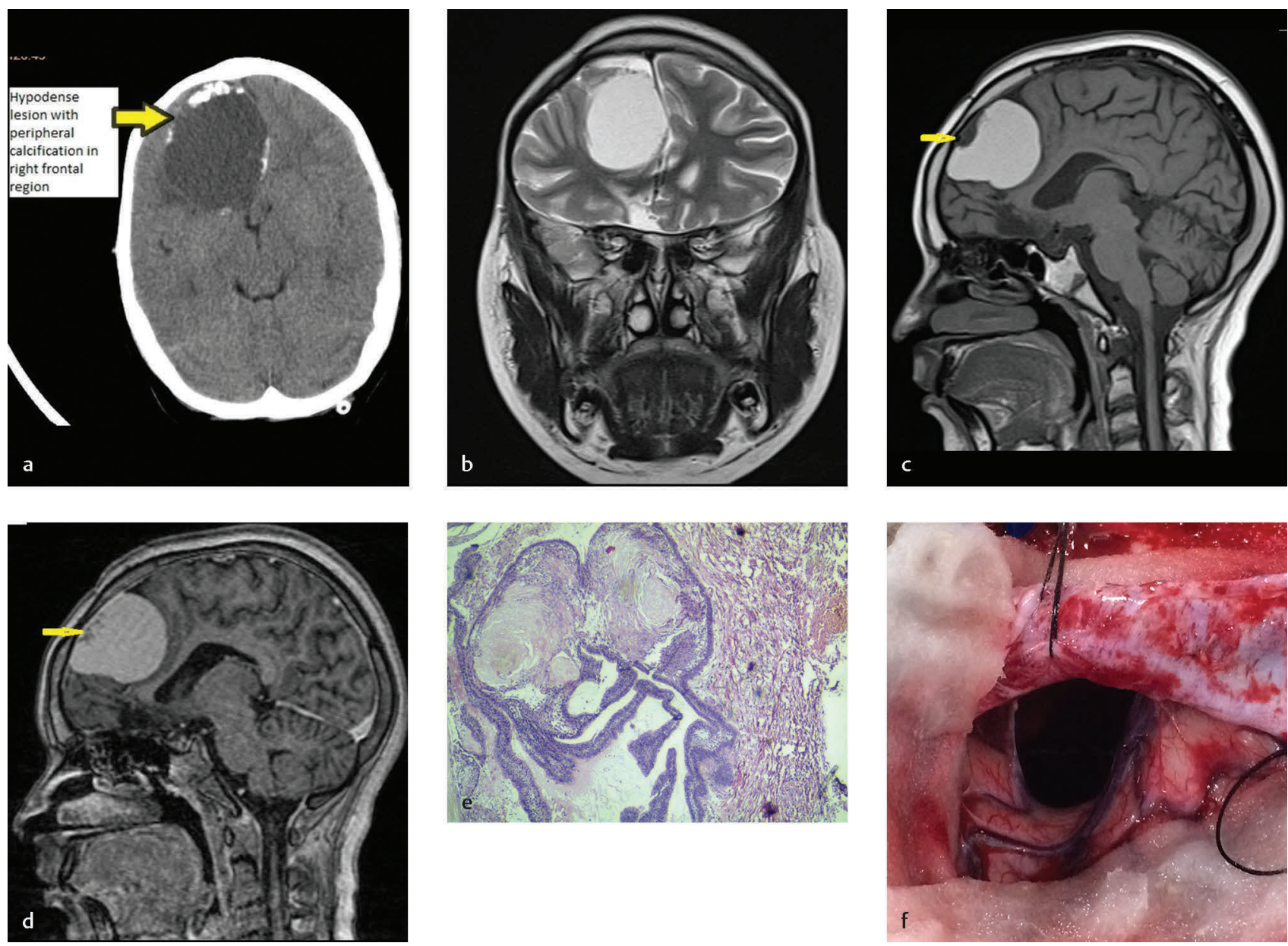

Fig. 2 (a) NCCT showing well-defined, extra-axial, hypodense lesion with discontinuous rim of calcification in right frontal region with mass effect. (b) T2 coronal MRI shows heterogeneously hyperintense lesion in parasagittal location in right frontal region. (c) T1 sagittal MRI shows heterogeneously hyperintense lesion in extra-axial location in right frontal region. (d) T1 sagittal postcontrast MRI showing heterogeneously enhancing solid area within lesion. (e) Intraoperative image of the tumor cavity. (f) HPE 40x. Complex squamous epithelium with stellate reticulum. Microcyst and wet keratin are present with fibrous stroma.

is difficult to estimate as there are only a few reported cases. Before this case, 60 ectopic recurrent craniopharyngiomas have been reported up until 2016. ${ }^{5}$ There are two proposed theories for ectopic recurrence. The more common of the two is direct implantation of tumor cells along the operative tract. The second theory is the dissemination of tumor cells through CSF to ectopic location. Nomura et al were able to confirm tumor seeds in CSF following surgery, and two separate ectopic lesions were attributed to both mechanisms in their case. Out of the 60 cases, 33 tumors were located in the previous surgical routes and 27 were disseminated along the cerebrospinal fluid pathway, which was almost equal (surgical tract 55\%, CSF $45 \%$ ). All reported cases underwent surgery. ${ }^{13,5-7}$ Ectopic recurrence can occur over a wide range of time after resection (1 month-26 years), with a median of 4 years. Equal prevalence was seen in male and female patients ( $43 \%$ female, $57 \%$ male). ${ }^{8}$ Children and adult patients were equally affected ( 27 vs. 24 cases). Male children were affected predominantly (62\%). ${ }^{1}$

The frontal lobe is the most common site of ectopic recurrence (23 cases). Epidural and sylvian fissures are also common sites where implantation via surgical route is the predominant mechanism. Location of ectopic recurrent craniopharyngioma in the posterior fossa, spinal cord, cerebellopontine angle, and prepontine cisterns was strictly associated with CSF contamination by tumor cells. ${ }^{9}$ Extra-axial parasagittal location in the frontal region has not been reported before this case. ${ }^{1} \mathrm{~A}$ variety of techniques in surgery have been proposed to prevent recurrence. However, these techniques may not always be successful. It was found that most reported ectopic recurrent tumors had some cystic component that was also present in primary tumor. Presence of cystic component in primary lesion is a major deciding factor for ectopic recurrence after surgery of primary tumor. ${ }^{10}$ The reason for this could be the higher chance of the cystic part to break easily during surgical manipulation with release of its contents into the surrounding areas/CSF. In our case too, the ectopic tumor had cystic component., ${ }^{2,3}$ Vascular endothelial growth factor and its receptor are reported to be significantly more expressed for ectopic recurrences than for the primary lesion. Tumor cells showed positive immune-histochemical staining of up to $33 \%$, whereas in the primary tumor neither 
vascular endothelial growth factor nor its receptor could be detected on tumor cells. However, the role of angiogenesis in ectopic recurrence of craniopharyngioma has yet to be established. ${ }^{1,11}$ There is a need for more research to determine the exact cause of ectopic recurrence of craniopharyngioma. ${ }^{1}$ Extra caution needs to be exercised while operating on cystic craniopharyngioma to avoid spillage of its contents. Some authors approve the use of cotton for isolation of the tumor and aspiration of cystic contents prior to dissection of capsule. Long-term clinical and radiologic follow-up of the patients is recommended. ${ }^{2,3}$

Elliott et al reported about the treatment of 31 cases of ectopic recurrence. Twenty-two patients underwent GTR that provided good disease control as seen on follow-up visits. In this series, the underlying factors for subtotal resection of ectopic recurrences included recurrence involving the brainstem and deep white matter as well as significant medical comorbidities contraindicating extensive surgical resection. If the recurrence is primarily cystic and in a surgically accessible region of the brain, GTR remains the primary modality of treatment as in our case. However, where GTR is not feasible, gamma knife radiotherapy, intracavitary irradiation, or chemotherapy with bleomycin or interferon can be alternative modalities of treatment. ${ }^{15,12}$ Ectopic recurrence of craniopharyngioma via CSF/surgical tract is a rare possibility that needs to be addressed during surgery of primary tumor. It is important to remove primary lesion in toto. Cystic tumors need special attention during removal because of their high recurrence rate.

Conflict of Interest

There is no conflict of interest.

\section{References}

1 Jakobs M, Orakcioglu B. Ectopic recurrent craniopharyngioma of the frontal bone. J Neurosurg 2012;117(3):490-497

2 Fahlbusch R, Honegger J, Paulus W, Huk W, Buchfelder M. Surgical treatment of craniopharyngiomas: experience with 168 patients. J Neurosurg 1999;90(2):237-250

3 Mortini P, Gagliardi F, Boari N, Losa M. Surgical strategies and modern therapeutic options in the treatment of craniopharyngiomas. Crit Rev Oncol Hematol 2013;88(3):514-529

4 Louis DN, Ohgaki H, Wiestler OD, et al. The 2007 WHO classification of tumours of the central nervous system. Acta Neuropathol 2007;114(2):97-109

5 Du C, Feng CY, Yuan J, Yuan X. Ectopic recurrence of pediatric craniopharyngiomas after gross total resection: a report of two cases and a review of the literature. Childs Nerv Syst 2016;32(8):1523-1529

6 Gupta DK, Ojha BK, Sarkar C, Mahapatra AK, Sharma BS, Mehta VS. Recurrence in pediatric craniopharyngiomas: analysis of clinical and histological features. Childs Nerv Syst 2006;22(1):50-55

7 Yang Y, Shrestha D, Shi XE, Zhou Z, Qi X, Qian H. Ectopic recurrence of craniopharyngioma: reporting three new cases. $\mathrm{Br}$ J Neurosurg 2015;29(2):295-297

8 Boongird A, Laothamatas J, Larbcharoensub N, Phudhichareonrat S. Malignant craniopharyngioma; case report and review of the literature. Neuropathology 2009;29(5):591-596

9 Nomura H, Kurimoto M, Nagai S, et al. Multiple intracranial seeding of craniopharyngioma after repeated surgery-case report. Neurol Med Chir (Tokyo) 2002;42(6):268-271

10 Zhao X, Yi X, Wang H, Zhao H. An analysis of related factors of surgical results for patients with craniopharyngiomas. Clin Neurol Neurosurg 2012;114(2):149-155

11 Garrè ML, Cama A. Craniopharyngioma: modern concepts in pathogenesis and treatment. Curr Opin Pediatr 2007;19(4):471-479

12 Gao S, Shi X, Wang Y, Qian H, Liu C. Malignant transformation of craniopharyngioma: case report and review of the literature. J Neurooncol 2011;103(3):719-725 